\title{
AN ARC OF FINITE 2-MEASURE THAT IS NOT RATIONALLY CONVEX
}

\author{
THOMAS BAGBY AND P. M. GAUTHIER
}

(Communicated by Clifford J. Earle, Jr.)

ABstract. A subset of $\mathbf{C}^{2}$ having the properties in the title is constructed.

If $K$ is a compact subset of $\mathbf{C}^{n}$, let $\pi(K)$ and $\rho(K)$ denote the polynomially convex hull of $K$ and the rationally convex hull of $K$, respectively. We then have $\pi(K) \supset \rho(K) \supset K$. If $K$ has zero two-dimensional Hausdorff measure, it is well known that $\rho(K)=K$. If $\Gamma$ is a compact Jordan arc (homeomorph of $[0,1])$ with finite one-dimensional Hausdorff measure, a deep result of Alexander [A] states that $\pi(\Gamma)=\Gamma$. Thus Gamelin [G] has asked the following questions:

(1) If $\Gamma$ is a compact Jordan arc in $\mathbf{C}^{n}$ that has finite two-dimensional Hausdorff measure, does it follow that $\rho(\Gamma)=\Gamma$ ?

(2) If $\alpha \in(1,2]$ and $\Gamma$ is a compact Jordan arc in $\mathbf{C}^{n}$ that has finite $\alpha$-dimensional Hausdorff measure, does it follow that $\pi(\Gamma)=\Gamma$ ?

The purpose of this note is to point out that question 1 can be answered by combining a recent theorem of $\mathrm{Uy}[\mathrm{U}]$ with a well-known construction of Wermer [W] and Rudin [R]. This also gives an answer to question 2 in case $\alpha=2$.

Theorem. If $n \geq 2$, there exists a compact Jordan arc $\Gamma \subset \mathbf{C}^{n}$ that has finite two-dimensional Hausdorff measure, but $\rho(\Gamma) \neq \Gamma$. (In particular, $\pi(\Gamma) \neq \Gamma$.)

Proof. We may assume $n=2$. Let $E$ be a totally disconnected compact subset of $\mathbf{C}$ with positive two-dimensional Lebesgue measure. From the theorem of Uy [U] there exists a bounded Lipschitz function $f: \mathbf{C} \rightarrow \mathbf{C}$ such that the restriction $\left.f\right|_{\mathbf{C} \backslash E}$ is holomorphic and nonconstant, and $f(\infty)=0$. We define $M: \overline{\mathbf{C}} \rightarrow \mathbf{C}^{2}$ by $M(z) \equiv(f(z), z f(z))$, and note that $M$ is injective on the complement of $f^{-1}(0)$. Then the image $X=M(E)$ is totally disconnected (in fact, if $Y$ denotes the quotient space obtained from $E$ by identifying all points of $E \cap f^{-1}(0)$, then $Y$ is totally disconnected and $M$ induces a homeomorphism from $Y$ onto $X$.) It then follows from a theorem of Antoine [An] that there is a Jordan arc $\Gamma \subset \mathbf{C}^{2}$ containing $X$ such that $\Gamma \backslash X$ is locally polygonal. This property, and the fact that $M$ is Lipschitz on $E$, show that the set $\Gamma=$

Received by the editors October 23, 1990.

1980 Mathematics Subject Classification (1985 Revision). Primary 32E30; Secondary 32E20.

Research was supported by NSERC of Canada and FCAR du Québec. 
$(\Gamma \backslash X) \cup M(E)$ has finite two-dimensional Hausdorff measure. Now using the fact that the restriction of $M$ to $W=\mathbf{C} \backslash\left[E \cup f^{-1}(0)\right]$ is an immersion, while the set $\Gamma \backslash X$ is locally rectifiable, we see that the set $M(W) \backslash(\Gamma \backslash X)=M(W) \backslash \Gamma$ is nonempty; we select a point $\omega \in W$ such that $M(\omega) \notin \Gamma$.

To complete the proof of the theorem, it suffices to show that $M(\omega) \in \rho(\Gamma)$. If not, it is well known that there is a polynomial $p$ on $\mathbf{C}^{2}$ that vanishes at $M(\omega)$ but never on $\Gamma$. Then $g \equiv p \circ M: \overline{\mathbf{C}} \rightarrow \mathbf{C}$ is continuous and $\left.g\right|_{\overline{\mathbf{C}} \backslash E}$ is holomorphic. Moreover, $g$ never vanishes on $E$, so $g$ vanishes in only finite many points of $\mathbf{C} \backslash E$; let these points be denoted (according to multiplicity) by $\zeta_{1}=\omega, \zeta_{2}, \ldots, \zeta_{r}$, where $r \geq 1$. Then $h(z) \equiv g(z) /\left(z-\zeta_{1}\right) \cdots\left(z-\zeta_{r}\right)$ never vanishes on $\mathbf{C}$, so there exists a continuous function $H: \mathbf{C} \rightarrow \mathbf{C}$ such that $h \equiv e^{H}$ on $\mathrm{C}$. We have now obtained a deleted neighborhood of infinity, $C \backslash E$, on which there are holomorphic functions $h$ and $H$, such that $h \equiv e^{H}$ and $h(\infty)=0$; this is impossible, so the theorem is proved.

\section{REFERENCES}

[A] H. Alexander, Polynomial approximation and hulls in sets of finite linear measure in $\mathbf{C}^{n}$, Amer. J. Math. 93 (1971), 65-74.

[An] L. Antoine, Sur la possibilité d'étendre l'homéomorphie de deux figures à leur voisinage, $\mathrm{C}$. R. Acad. Sci. Paris Sér. I Math. 171 (1920), 661-663.

[G] T. W. Gamelin, Polynomial approximation on thin sets (Symposium on Several Complex Variables, 1970), Lecture Notes in Math., vol. 184, Springer-Verlag, Berlin, 1970, pp. 5078.

[R] W. Rudin, Subalgebras of spaces of continuous functions, Proc. Amer. Math. Soc. 7 (1956), 825-830.

[U] Nguyen Xuan Uy, Removable sets of analytic functions satisfying a Lipschitz condition, Ark. Mat. 17 (1979), 19-27.

[W] J. Wermer, Polynomial approximation on an arc in $\mathbf{C}^{3}$, Ann. of Math. (2) 62 (1955), 269-270.

Department of Mathematics, Swain Hall-East, Indiana University, Bloomington, INDIANA 47405

Départment de Mathématiques et de Statistique, Université de Montréal, C. P. 6128 , Succ. "A", Montréal, P. Q. H3C 3J7, Canada 